\title{
INTERDISCIPLINARIDADE DOS CURSOS DA ÁREA DA SAÚDE DA UPF EM RELAÇÃO ÀS INTERVENÇÕES PRÁTICAS NA EQUOTERAPIA
}

\author{
Paulo Cezar Mello ${ }^{1}$, Nadir Antônio Pilcher ${ }^{2}$ e Helenice de Moura Scortegagna ${ }^{2}$ \\ ${ }^{1}$ UPF - Universidade de Passo Fundo, FEFF - Faculdade de Educação Física e Fisioterapia \\ ${ }^{2}$ PPGEH - Programa de Pós Graduação Strictu Senso Envelhecimento Humano
}

pcmello@upf.br

Acesso DOI: http://dx.doi.org/10.34059/ciejop.2019v28i1-4

\begin{abstract}
Resumo
MELLO, P.C; PILCHER, N.A. e SCORTEGAGNA, H.M. Interdisciplinaridade dos cursos da área de saúde da UPF em relação às intervenções práticas na equoterapia. Revista Científica JOPEF, Vol.28, n.1, pp.44-53, 2019. O projeto de Equoterapia ocorre de maneira interdisciplinar e multidisciplinar onde participam em média cinquenta acadêmicos bolsistas paidex e voluntários dos cursos área da saúde como Educação Física, Enfermagem, Fonoaudiologia, Odontologia, Psicologia e Medicina Veterinária, proporcionando uma interdisciplinaridade entre alunos/pacientes atendidos e transdisciplinaridade estabelecendo conexões entre os cursos, buscando experiências significativas vivenciadas nas diversas situações de construção de conhecimentos vinculados a realidade social com as quais irão se defrontar no futuro exercício profissional nas diferentes áreas da saúde. As sessões de equoterapia são realizadas nas segundas e quartas-feiras, pelo turno da tarde, na Fazenda da Brigada Militar. Os sujeitos atendidos neste projeto são indivíduos com deficiências, em sua maioria com transtorno do espectro autista e paralisia cerebral, todos pertencentes à comunidade de Passo Fundo. No total estão sendo atendidas semanalmente uma média trinta pessoas com necessidades especiais, com a faixa etária que variam de três aos quarenta e três anos de idade. No decorrer das atividades, tem sido possível observar o desenvolvimento neuropsicomotor com enorme melhora na coordenação, ritmo, equilíbrio, postura e autoconfiança, possibilitando maior independência e autonomia dos atendidos. Atualmente, três profissionais conduzem o projeto, um professor coordenador da Universidade de Passo Fundo, lotado na Faculdade de Educação Física e Fisioterapia, outros dois colaboradores nas áreas de equitação e cuidados com os equinos. Este projeto é realizado em parceria com Associação dos Pais e Amigos dos Autistas (AUMA), Centro de Atendimento Socioeducativo (CASE) e $3^{\circ}$ RPMON da Brigada Militar.
\end{abstract}

Palavras-chave: Equoterapia. Transtorno do Espectro Autista. Paralisia Cerebral.

\begin{abstract}
MELLO, P.C; PILCHER, N.A. e SCORTEGAGNA, H.M. Interdisciplinaridade dos cursos da área de saúde da UPF em relação às intervenções práticas na equoterapia. Revista Científica JOPEF, Vol.28, n.1, pp.44-53, 2019. The Equine Therapy project takes place in a
\end{abstract}

Fórum Internacional de Qualidade de Vida e Saúde - Balneário Camboriú/SC, 12 de outubro de 2019 
unique and interdisciplinary and multidisciplinary way in which fifty paidex scholarship students and volunteers from health courses such as Physical Education, Nursing, Speech Therapy, Dentistry, Psychology and Veterinary Medicine participate, providing an interdisciplinarity between students / patients treated and transdisciplinarity. establishing connections between the courses, seeking meaningful experiences lived in different situations of knowledge construction linked to the social reality that they will face in the future professional practice in different areas of health. Equine therapy sessions are held on Monday and Wednesday, by the afternoon shift, at the Military Brigade Farm. The subjects assisted in this project are individuals with disabilities, mostly with autism spectrum disorder and cerebral palsy, all belonging to the Passo Fundo community. In total, an average of thirty people with special needs are being served weekly, ranging in age from three to forty-three years old. During the activities, it has been possible to observe the neuropsychomotor development with huge improvement in coordination, rhythm, balance, posture and selfconfidence, allowing greater independence and autonomy of those cared for. Currently, three professionals lead the project, a coordinating professor at the University of Passo Fundo, based at the Faculty of Physical Education and Physiotherapy, two other collaborators in the areas of horse riding and care. This project is carried out in partnership with the Autistic Parents and Friends Association (AUMA), Socioeducative Care Center (CASE) and the 3rd RPMON of the Military Brigade.

Keywords: Equine Therapy. Autistic Spectrum Disorder. Cerebral Palsy.

\section{Introdução}

Projeto de extensão denominado Educação Inclusiva Equoterapêutica visa estimular a parte motora e biopsicossocial de pessoas com espectro autista e paralisia cerebral, além da ambientação ecológica, respeito aos animais e à vida. Nesse sentido, analisa-se 0 contexto com abordagem interdisciplinar e multiprofissional, por meio da construção da autoimagem e da cidadania de pessoas com deficiência tendo como objetivo disponibilizar na área específica de Equoterapia recursos terapêuticos e pedagógicos para potencializar a qualidade de vida de pessoas ou de grupos em situação de vulnerabilidade social.

As pessoas atendidas são alunos/pacientes em processo de aprendizagem, neste sentido, não são vistas como um deficiente ou identificadas por uma patologia da qual são portadoras. Cada pessoa apresenta características próprias, ou seja, é uma singularidade na coletividade e coletividade que se faz nas singularidades. As intervenções são vivenciadas como um processo de aprendizagem onde todos os envolvidos são aprendizes e mestres simultaneamente. Entretanto, nem todos aprendem as mesmas questões ao mesmo tempo. Diante disso, ressalta a opção por uma forma de atendimento no qual, adultos e crianças são estimulados a realizar as atividades com ênfase na expressão lúdica, ou seja, com alegria, prazer, divertimento, jogos e brincadeiras que eliminam a característica clínica hospitalar da 
terapia convencional, em relação ao cuidado, buscando desta forma, resultados altamente positivos na melhoria da qualidade de vida destas populações.

Tendo como objetivo potencializar a qualidade de vida dos praticantes, conceitua-se a "Equoterapia como um método terapêutico que utiliza o cavalo dentro de uma abordagem interdisciplinar nas áreas de saúde, educação e equitação, buscando o desenvolvimento biopsicossocial de pessoas com deficiência e/ou com necessidades especiais" (ANDE-BRASIL, 2006).

\section{Diagnóstico da realidade}

O projeto de Equoterapia ocorre em parceria com a Associação de Pais e Amigos dos Autistas (AUMA), Centro de Atendimento Socioeducativo (CASE) e Brigada Militar. No total estão sendo atendidas em média trinta pessoas com necessidades especiais, com idades entre os três aos quarenta e três anos. As atividades são oferecidas sistemática e gratuitamente, com resultados altamente positivos. As sessões são realizadas em grupos, de até no máximo cinco cavaleiros simultaneamente, as segundas e quartas-feiras, pela parte da tarde, na Fazenda da Brigada Militar.

Essa opção encontra respaldo nos novos paradigmas interpretativos da realidade, com destaque para o pensamento complexo de Morin (1991), cuja tese central sustenta ser o homem a um só tempo biológico, cultural e social; para a Teoria Ecológica de Capra (1994) o qual afirma que a vida é constituída de uma ampla e complexa rede de relações (teia da vida) muitas das quais ocultas e/ou desconhecidas. Kuhn (1991), afirma ainda, que o paradigma se constitui uma rede de compromissos ou adesões conceituais, teóricas e metodológicas por meio de instrumentais compartilhados. Tomando por base estes pressupostos teóricos, os quais trazem avanços significativos em relação aos paradigmas tradicionais sobre a compreensão dos fenômenos saúde/doença/tratamento, a metodologia proposta para as atividades de Equoterapia que são realizadas de forma coletiva.

Com base em conhecimentos teóricos e científicos, foi desenvolvido protocolo interdisciplinar de atividades que iniciam pela aproximação e familiarização com o ambiente, com a equipe e com os cavalos. Acolhimento, estabelecimento de vínculo, acompanhamento dos projetos terapêuticos, que são diretrizes que para se 
efetivarem na rede de serviços, como mudanças estruturais na produção do cuidado, são dependentes das tecnologias leves (FRANCO E MERHY, 2012). Considerando que ao final de cada dia de trabalho é realizada reunião com os acadêmicos estagiários e professor no sentido de anotar e comentar aspectos relativos ao desenvolvimento das sessões.

\section{Metodologia}

Esta pesquisa caracteriza-se como descritiva observacional, envolvendo crianças, jovens e adultos com Transtorno do Espectro Autista e Paralisia Cerebral praticantes de projeto de extensão em Educação Inclusiva Equoterapêutica.

O local da pesquisa foi na cidade de Passo Fundo (RS), na Fazenda da Brigada Militar de Passo Fundo. A amostra foi realizada com 30 crianças, adolescentes e adultos de 03 a 43 anos, totalizando 23 pessoas com Transtorno do Espectro Autista e 07 com Paralisia Cerebral $O$ estudo foi realizado durante o período de março de 2019 a setembro de 2019. O projeto é institucionalizado pela Universidade de Passo Fundo e pela Faculdade de Educação Física e Fisioterapia, e ocorrem de maneira sistemática desde 2013 com fluxo contínuo na UPF.

O projeto de Educação Inclusiva Equoterapêutica ocorre por meio de intervenção clínica pedagógica baseada nas teorias dos novos paradigmas. Segundo Kuhn (1991), os paradigmas são realizações científicas universalmente reconhecidas que, durante algum tempo, fornecem problemas e soluções modelares para uma comunidade de praticantes de uma ciência. A equoterapia sob este olhar trabalha com vistas à construção de processos que envolvam agentes educativos/alunos, enquanto a equoterapia tradicional acontece na relação profissional/paciente. Os processos pedagógicos da equoterapia aqui explicitada constituem-se de métodos e relações que facilitem a inserção e interação social assegurando as individualidades também com vistas à construção do bem estar do aluno/paciente.

As intervenções de equoterapia realizada pela equipe da UPF não é realizada em uma clínica ou hospital ou na própria casa, mas sim, em um ambiente revestido de contornos pedagógicos e lúdicos diversificado, especialmente pensando e concretizado objetivando a promoção da sensação de bem estar que é de fundamental importância no processo de envolvimento de novas aprendizagens de Fórum Internacional de Qualidade de Vida e Saúde - Balneário Camboriú/SC, 12 de outubro de 2019 
um aluno/paciente, diferenciando-se da ideia terapêutica, que objetiva a recuperação de um paciente.

$\mathrm{Na}$ perspectiva da equoterapia apresentada os alunos/pacientes atendidos utilizam uma montaria individual ou em dupla numa interação com os demais acadêmicos e com os colaboradores. $O$ encontro com outros (semelhantes/diferentes) alunos, com a equipe interdisciplinar, cavalos, natureza, objetiva desenvolver 0 interesse de aprender e a vivenciar novas experiências. Nesse processo tudo é visto como causa e efeito ao mesmo tempo.

A concepção primária desta visão de equoterapia é a de que o participante ao controlar um animal de grande porte, forte e interativo, desenvolva e aumente sua autoconfiança. De que a sensação de ser capaz, de estar no comando, de poder realizar alguma coisa, fortaleça a sua autoestima, concretize atividades que para muitas pessoas, crianças ou adultos, nem sempre parecem coisas fáceis e corriqueiras. Montado em um cavalo o aluno/paciente modifica seu referencial de mundo, aumentando a sua amplitude, sua relação com os outros, possibilitando novas construções e interesses para com o mundo exterior.

A relação de aceitação pelos outros contribui de forma significativa para reduzir as sensações de angústia, medo e insegurança frente a um mundo diferente de seu cotidiano. Essas relações se revestem de maior importância, especialmente para iniciantes. O cavalo é, comumente, uma novidade em relação a outras atividades já vivenciadas. Por ser um animal dócil e cordato, favorece o estabelecimento de relações de confiança muito rapidamente. A presença da equipe multidisciplinar também é fator determinante no processo de vivenciar novas e diferentes situações e percepções de modo seguro e controlado no que diz respeito à segurança e confiança.

Cabe ressaltar que a equipe multidisciplinar composta pelos acadêmicos estagiários (Educação Física, Fisioterapia, Enfermagem, Fonoaudiologia, Odontologia, Psicologia e Medicina Veterinária) possibilitarem ao aluno/paciente experimentar situações em que se sintam, ao mesmo tempo, ameaçado/encorajado/desafiado, como na maioria das situações da vida. A construção de situações comuns pode criar uma relação de troca colaborando nos resultados e metas da equoterapia.

O trabalho coletivo, em especial com os autistas pode favorecer a construção de novos comportamentos e interações que levem a experimentar novos papéis que Fórum Internacional de Qualidade de Vida e Saúde - Balneário Camboriú/SC, 12 de outubro de 2019 
contribuem para "sair de si mesmos", a aceitar e se deixar aceitar. Ao observarem os colegas e os acadêmicos voluntários, os alunos/pacientes tendem a modificar seus próprios comportamentos, que serão observados pelos outros e, assim, constituindo uma circularidade que retroalimenta todo o processo de ensino/aprendizagem com efetividade de todos enquanto sujeitos.

As atividades coletivas de equoterapia proporcionam ao aluno/paciente a vivência de sua interação como indivíduo no grupo e no mundo. Ao fazer parte de um grupo o indivíduo adquire novos papéis que Ihe seria mais difícil desenvolver e atuar em atividades individuais. No processo de exteriorização da individualidade que ocorre nas atividades em grupo, se constrói novas realidades e novas maneiras de vivenciá-las.

Os novos comportamentos que apresentam tem forte relação com a metodologia utilizada que deverá privilegiar as manifestações de afeto e cooperação entre os alunos/pacientes, em relação à capacidade de comunicação, ao companheirismo, ao respeito, ao interesse na continuidade da atividade, à auto iniciativa, à autoconfiança, respeito para consigo e com os outros, à diminuição da ansiedade, à superação de problemas, à aceitação do diferente, ao aumento da tolerância a frustração e aos seus limites e reconhecimento de suas possibilidades, coragem, disposição.

As sessões são realizadas em grupos de até no máximo cinco cavaleiros simultaneamente. $O$ ambiente onde são realizadas as atividades (Fazenda da Brigada Militar) é organizado com características lúdicas tendo presente que a maioria dos frequentadores é de crianças e adolescentes. Por isso está repleto de estímulos visuais de diferentes naturezas, relacionados ao mundo imaginário, colocados em posições estratégicas para que os praticantes observam o que está abaixo, acima e aos lados, tais como pneus coloridos, arcos, cestas, bolas de diversos tamanhos, texturas e cores entre outros.

Enquanto parte da equipe de colaboradores e estagiários conduzem as atividades, outros permanecem em contato direto com os acompanhantes. Nessas "conversas informais" são observados detalhes da percepção destes sobre a evolução do aluno/paciente, sua rotina e o cotidiano familiar. A metodologia de trabalho utilizada facilita a constante interação entre alunos/alunos, alunos/estagiários, alunos/ambiente considerando o "clima" de descontração, alegria e bem-estar. Ao se dirigir ao local da atividade equoterapêutica permite que o Fórum Internacional de Qualidade de Vida e Saúde - Balneário Camboriú/SC, 12 de outubro de 2019 
aluno/paciente montado no cavalo interaja com a equipe interdisciplinar, com os cavalos, a natureza etc., sendo estimulado a aprender e a vivenciar novas experiências.

As atividades de Equoterapia são realizadas com a presença de profissionais e acadêmicos estagiários dos cursos da área da saúde da UPF, com duração de aproximadamente 25 a 30 minutos, sendo o aluno/paciente montado, acompanhado lateralmente por dois assistentes (estagiários) e um condutor de guia do cavalo (adolescente CASE), realizadas quando possível pelas condições meteorológicas, já que as atividades são realizadas a céu aberto.

As sessões são realizadas dentro de uma campo aberto, de forma coletiva, iniciando com o aluno/paciente montado, na andadura ao passo, fazendo um trajeto pré-direcionado. Um professor responsável orienta o desenvolvimento das atividades e observa atentamente cada aluno/paciente, motivando-o na realização das tarefas e prestando apoio e segurança. No percurso são propostas atividades lúdicas, como os jogos com bolinhas de plástico, alinhavos, encaixes, petecas, bolas de diferentes tamanhos e texturas, cestas coloridas e/ou alvos, pneus, bichos de fantoches etc. com a finalidade de entretenimento e diminuição de ansiedade enquanto são realizados exercícios fisioterápicos e fonoaudiólogos. Os limites e as possibilidades de cada aluno/paciente são sempre respeitados. Estímulos verbais e visuais são trabalhados o tempo todo incentivando o aluno/paciente a desenvolver potencialidades. Isso faz com que ele sinta-se motivado a fazer os exercícios sugeridos tais como movimentos de flexão e extensão de membros superiores e inferiores sendo trabalhados conforme as limitações e potencialidades de cada participante.

Estas atividades são realizadas dentro de uma proposta lúdica, divertida e prazerosa, através de recursos como cantos de músicas de seu conhecimento, historinhas divertidas, reconhecimento de palavras, letras, números e distinção de cores. A realização das sessões não segue um plano prévio rígido ou engessado em sequência monótona, mas sim, conforme o interesse, disposição, humor, estado de saúde apresentada pelo aluno/paciente na hora da atividade e também das condições climáticas. No inicio e final das atividades os participantes são estimulados a cumprimentar, agradecer, a "falar" com e acariciar os animais. São recebidos alegremente pela equipe multidisciplinar a qual cumprimenta seus 
acompanhantes laterais, tanto na chegada como na partida, procurando com isso ampliar o contato afetivo e de incentivo ao retorno na próxima aula.

Após as atividades a equipe realiza reunião de avaliação onde são examinados todos os fatos ocorridos nas sessões. Também são motivos de reflexão e análise os comportamentos das pessoas atendidas bem como manifestações dos pais e acompanhantes, constituindo assim, dados para o relatório diário da atividade e continuidade dos atendimentos.

Em dias e horários definidos previamente, os acadêmicos bolsistas devem participar das reuniões de planejamento onde são definidas as estratégias das atividades, escolhas dos materiais e procedimentos pedagógicos necessários. Também, ao final de cada semestre são realizadas reuniões com pais e/ou responsáveis, a fim de apresentar e comentar aspectos relativos ao desenvolvimento individual de cada aluno/paciente.

\section{Análise e discussão dos resultados}

No decorrer das atividades tem sido possível observar desenvolvimento neuropsicomotor com enorme melhora na coordenação, ritmo, equilíbrio, postura, autoconfiança. Foram observadas situações de sucesso na realização de movimentos mais complexos, relativos à autoimagem, sendo apontados ainda melhora no sono, disposição geral, interesse para ampliar repertório de conhecimentos, maior sociabilização e iniciativas buscando independência e autonomia.

Para além de dados quantitativos ou de número de atendimentos, os depoimentos de pais e cuidadores das pessoas que frequentam as sessões de Equoterapia em busca de tratamento, refletem os resultados e ganhos obtidos em aspectos relativos à potencialização da qualidade de vida. Afirmam eles: "a ansiedade e o desejo das crianças de verem se aproximar o dia de equo é um sinalizador do interesse e gosto pelas atividades". Os acadêmicos que participam das atividades demonstram ganhos de conhecimento ao aprenderem a lidar e criar metodologias de trabalho adequadas ao perfil dos participantes. Segundo seus relatos: "aprendem a compreender os comportamentos dos alunos/pacientes atendidos, os determinantes que os levam a situação em que se encontram demonstrando tolerância e respeito em relação a isso". Cabe destacar a participação 
efetiva dos adolescentes do CASE no trabalho de condutores dos cavalos. As observações e acompanhamento dos agentes socioeducadores em relação ao envolvimento destes jovens na realização dos trabalhos é um fato de significativa relevância. Os mesmos são cooperativos, demonstram profundo respeito pelos alunos/pacientes atendidos, participando ativamente na organização do local para a realização das atividades. Em situações específicas, demonstram iniciativa e atenção criando condições apropriadas de prevenção a qualquer possibilidade de acidente ou situação complexa.

Diante disso, adotou-se como critério de observação em relação a depoimentos e verbalizações dos participantes, familiares, junto com informações prestadas pelos médicos responsáveis pelo encaminhamento e aspectos gerais apresentados e discutidos em reuniões avaliativas, relativos ao ajuste tônico, coordenação motora geral e fina, coordenação óculo-manual, equilíbrio, atenção, ritmo; compreensão e êxito na realização das atividades propostas, e aspectos relativos à autoestima, independência, autonomia, confiança em si e nos demais, relações e interações com colegas e terapeutas, interação com o cavalo.

\section{Conclusão}

A ideia de fazer equoterapia utilizando os conhecimentos da equipe multidisciplinar compreende-se que possui relevância tanto para o aluno/paciente que ganha nas terapias com uma abordagem diferente que a usual na clínica, quanto para os acadêmicos estagiários, que adquirem um saber prático em um ambiente não comum a sua realidade. A maneira como a terapia tem um fluxo, dependendo do perfil do aluno/paciente que vai além de sua deficiência, do cavalo, do maior espaço físico, do clima em relação ao tempo, faz com que os métodos mudem a todo o momento, porém que os resultados continuem os mesmos, priorizando o bem estar e a melhoria de cada aluno/paciente.

A ação provocativa do descobrir constante e de valorização do conhecimento ampliam as expectativas frente aos novos desafios impostos pelas características das atividades contribuindo para a superação de limites. Para além de dados quantitativos ou de número de atendimentos, os depoimentos de pais, colaboradores e cuidadores das pessoas que frequentam as sessões de Equoterapia em busca de 
tratamento, refletem os resultados e ganhos obtidos em aspectos relativos à potencialização da qualidade de vida das pessoas com deficiência e vulnerabilidade social.

\section{Referências}

ASSOCIAÇÃO ARGENTINA DE ACTIVIDADES ECUESTRES PARA DISCAPACITADOS. Curso Avançado em Equinoterapia nível, II y III. Buenos Aires, Argentina, 2007.

ASSOCIAÇAO NACIONAL DE EQUOTERAPIA, n.13, jun./dez., 2006.

CAPRA, Fritjof. O ponto de mutação. São Paulo: Cultrix, 1994.

FRANCO, Túlio Batista e MERHY, Emerson Elias. Cartografias do Trabalho e Cuidado em Saúde. Revista Tempus Actas de Saúde Coletiva. Pág. 151 - 163. 2012.

FREIRE, Heloisa Bruna. Equoterapia: experiências com crianças autistas. São Paulo: Vetor, 1999.

KUHN, Thomas. S. A estrutura das revoluções científicas. São Paulo: Perspectiva, 1991.

LERMONTOV, Tatiana. Psicomotricidade na Equoterapia. São Paulo: Ideias e Letras, 2004.

MORIN, Edgar. Introdução ao pensamento complexo. Trad. Dulce Matos, Lisboa, 1991.

SANTOS, Sabrina Lombardi. Fisioterapia na Equoterapia. São Paulo: Ideias e Letras, 2005.

UZUN, Ana Luiza de Lara. Equoterapia: aplicação em distúrbios do equilíbrio. São Paulo: Vetor, 2005. 